\title{
ATLAS Upgrade Plans for the SLHC
}

\author{
J. Tseng ${ }^{\mathrm{a}}$

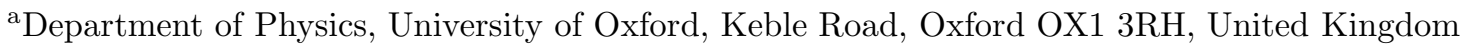 \\ The proposed luminosity upgrade to the LHC imposes significant challenges on the LHC detectors: their \\ design must function within a much harder radiation environment and yet preserve, if not improve, their ability \\ to maximize the upgrade's physics opportunities. In addition, they must be designed, constructed, and installed \\ on a tight timescale in order to be ready for operation in 2016. For ATLAS, the upgrade entails a major redesign \\ of the tracking systems, and possibly the forward calorimeters and muon detectors as well. Efforts have already \\ begun to address these issues.
}

\section{Introduction}

The ATLAS detector is a multi-purpose particle detector optimized for high- $p_{T}$ physics at the LHC. At its heart, in a $2 \mathrm{~T}$ solenoidal magnetic field, is the Inner Detector (ID), consisting of silicon pixels and strips as well as a Transition Radiation Tracker (TRT). These systems provide charged particle tracking in the pseudorapidity range $|\eta|<2.5$. Surrounding the tracker is electromagnetic and hadronic calorimetry covering $|\eta|<3.2$, with a liquid argon-based forward calorimeter extending the coverage to $|\eta|<4.9$. Outside the calorimeters is the muon spectrometer, which provides triggering and momentum measurement out to $|\eta|<2.7$. Details of the current detector configuration can be found in the ATLAS Technical Design Report [1].

A discussion of upgrade machine (SLHC) scenarios can be found elsewhere in these proceedings [2], as well as a summary of its physics potential [3]. In order the take advantage of the opportunities afforded by the increased luminosity, the aim of the ATLAS upgrade is to preserve, if not improve, the performance of the ATLAS detector in spite of the harsher radiation and background environment of the SLHC.

This article summarizes some of the issues and studies underway to address the challenges of pursuing physics at the SLHC with the ATLAS detector. An earlier and more comprehensive survey can be found in [4], with further develop- ments documented in [5]. As the machine upgrade has become better understood, however, upgrade plans have become more focused, with more detailed studies now beginning.

\section{Upgrade Parameters}

The Physics Opportunities and Future Proton Accelerators (POFPA) committee recommended a 50 ns bunch spacing configuration as the SLHC baseline [6]. If it can be implemented, this configuration would result in a peak instantaneous luminosity of $10^{35} \mathrm{~cm}^{-2} \mathrm{~s}^{-1}$, roughly 10 times the design luminosity of the LHC, and 400 pp interactions ("pileup") per beam crossing at the start of a spill. Approximately $3000 \mathrm{fb}^{-1}$ of integrated luminosity is to be delivered by the SLHC. The large pileup and integrated exposure are major experimental challenges, both from the perspective of detector performance as well as radiation tolerance.

The baseline scenario also possibly requires the installation of new $8 \mathrm{~m}$ long "slim" quadrupole magnets near the interaction region, probably between the forward muon wheels.

Along with the baseline configuration, a "backup plan", with a 25 ns bunch spacing, has been defined by POFPA. While it entails fewer pileup events (average 300), it also requires the placement of dipole magnets adjacent to the Inner Detector.

This article will focus on the major detector up- 
grades which are implied by the harsher radiation environment and higher detector occupancies. As may be expected, the detectors at low radii $r$ and large pseudorapidity $\eta$ are most affected, including the Inner Detector, forward calorimeter, and forward muon wheels. The barrel calorimeters and muon chambers, on the other hand, are left largely untouched. In addition, new detector electronics may be required for increased radiation hardness, and readout systems are being evaluated to cope with the bandwidth requirements expected of the higher detector granularities and occupancies being discussed.

\section{Muon Spectrometer}

The ATLAS muon spectrometer was designed with a safety factor of 5 over the expected cavern background rate; if the SLHC background rate is roughly 10 times this expectation, then most of the muon detector occupancies will remain below $30 \%$, except in the inner and middle endcap layers. High rate replacements are beginning to be developed. In addition, chamber ageing studies are also in progress, to assess their continued viability through SLHC data-taking.

The main uncertainty in muon upgrade planning is the actual cavern background, which is already reflected in the current safety factor. The "worst case scenario", with 50 times the rate expected for LHC, would put almost all of the muon chambers in the forward and endcap regions above the $30 \%$ threshold [7]. Measurements from LHC data-taking are therefore essential for more detailed upgrade plans. In addition, the effect of installing the quadrupoles between the outer muon wheels has not been estimated.

The effect of improved shielding in the highbackground scenario has been investigated, but the improvement in detector performance has been found to be marginal. On the other hand, a much larger impact, reducing background rates by a factor of 2 to 3 , can be made by installing a beryllium beampipe out to $\pm 16 \mathrm{~m}$ [8]; this option would be much more cost effective than replacing a large area of muon chambers.

\section{Forward Calorimeter}

The high radiation environment of the forward calorimeter (FCAL) dictate the use of inherently radiation-hard liquid argon technology, as well as very small gap sizes, down to $250 \mu \mathrm{m}$, in order to limit the build-up of space charge. Under SLHC conditions, even these measures may be insufficient, especially in the modules closest to the interaction point. Space charge effects are being investigated with the Protvino accelerator using small test modules with gap sizes down to $100 \mu \mathrm{m}$.

The forward modules face an additional problem due to beam heating. Estimates of energy deposition per beam crossing vary between 2.6 and $7 \mathrm{TeV}$ at $10^{34} \mathrm{~cm}^{-2} \mathrm{~s}^{-1}$. The higher estimate results in a heat load of approximately $40 \mathrm{~W} / \mathrm{m}^{2}$, the majority in the module closest to the interaction point. A simplified finite element analysis simulation has been employed to examine the effects of ten times this and other heat loads in the forward region; the maximum temperature is found in this model to be just above the boiling point of liquid argon at the calorimeter's operating pressure [9].

Again, measurements in the LHC are needed to determine the scale of the problem. If the pessimistic case is correct, or, for instance, convection is less effective at distributing heat within the modules, then it is imperative that cooling is improved in the forward calorimeter, or that the calorimeter is replaced.

Replacing forward calorimeter modules represents a serious logistical challenge, since it would require opening the endcap cryostats. Such an operation entails a long process of disassembly and reassembly, possibly extending to two or three years, which is longer than the time currently alloted for upgrade installation. Different options for avoiding this process are being investigated, among them the idea of installing a "warm" forward calorimeter module in front of the current innermost module. This space is currently occupied by beampipe pumps; it is the same space where the dipole magnet would be placed in the $25 \mathrm{~ns}$ beam spacing scenario. This new forward calorimeter module would take the 
brunt of the heat load, and would require new technology as well as additional shielding on its inner face, in order to protect the Inner Detector.

\section{Inner Detector}

It was evident early on that the Inner Detector would be replaced in its entirety: the TRT would experience prohibitively large occupancies, while radiation damage to the sensors and front-end electronics of the silicon strip and pixel subsystems would seriously degrade their performance. The current expectation is that the Inner Detector will become an all-silicon system. Increased granularity will help reduce detector occupancy and thus preserve its ability to recognize charged tracks and displaced vertices. Improved resolution along the beamline may also be necessary in order to distinguish between associated and unassociated features in a beam crossing (for instance, roughly one in every hundred collisions produces a $b \bar{b}$ pair at $\sqrt{s}=14 \mathrm{TeV}$. At the same time, it will be necessary to minimize the material profile of the Inner Detector: efforts are already underway to investigate increased service multiplexing (including serial powering) and different construction techniques. Three issues will be discussed here: the radiation environment; the $b$ layer, which is also scheduled to be replaced in 2012; and the overall Inner Detector layout.

\subsection{Radiation dose}

One of the consequences of replacing the TRT with silicon is the loss of its neutron-absorbing properties. A preliminary study, using the FLUKA simulation, has been made of the radiation dose in the Inner Detector region after $3000 \mathrm{fb}^{-1}$ [10]. As expected, at lower radii, the primary source of low-energy fluence is the interaction point itself, while neutron albedo from the surrounding material dominates at larger radii. Lining the calorimeter with $5 \mathrm{~cm}$ polyethylene moderator would reduce this to an acceptable level, as shown in Fig. 1.

The simulation was repeated to assess the situation of an iron plug filling the space in front of the forward calorimeter; the fluence increases by a factor of 2.7, reducing to approximately

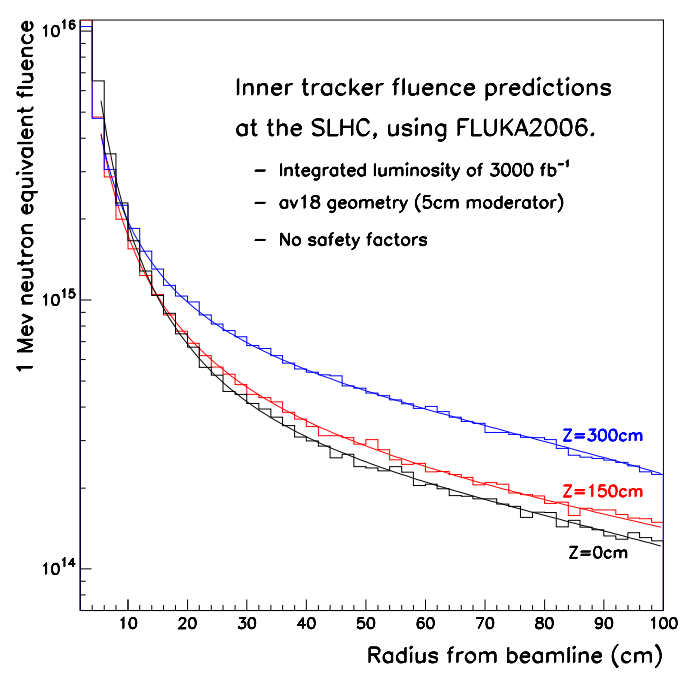

Figure 1. $1 \mathrm{MeV}$ neutron equivalent fluence predictions for the Inner Detector at $|z|=0,150$, and $300 \mathrm{~cm}$, using FLUKA simulation and implementing a $5 \mathrm{~cm}$ moderator.

2 if covered with polyethylene shielding. Compared to the two possible "fillers" - a new forward calorimeter module as described above, or, in the case of the 25 ns bunch spacing scenario, a dipole magnet - the iron plug is perhaps pessimistic, but the result suggests the need for caution in considering these possibilities.

\subsection{Pixel $b$ layer}

The innermost layer of pixels, the " $b$ layer", is subject to the harshest radiation conditions, and it is expected that current sensor technologies are insufficiently radiation hard for radii less than $5 \mathrm{~cm}$. New technologies are therefore being investigated which would allow sensors to be placed closer to the beampipe, which can have an outer diameter as small as $4.2 \mathrm{~cm}$.

Proposals have been submitted to ATLAS to study three-dimensional silicon sensors [11], thin silicon combined with three-dimensional interconnects [12], gas over slim silicon pixels (GOS- 
SIP) [13], and diamond semiconductors [14]. Prototypes have already been made and radiationtested for some of these sensor technologies.

It should be noted that the current $b$ layer is itself expected to be replaced around 2012, well before the main SLHC upgrade. If some of the technologies described in this section advance sufficiently quickly, they may be tested first on this timescale.

\subsection{Layout studies}

Fig. 2 shows the "strawman" Inner Detector layout which has been set as a starting point for evaluating the performance of different layout options. There are three distinct radial regions, corresponding to different sensor dimensions. The innermost barrel includes the " $b$ layer" at $r=5 \mathrm{~cm}$ along with 3 layers of pixels out to a radius of $28 \mathrm{~cm}$ and $|z|<40 \mathrm{~cm}$. The pixel size in the inner two layers is $50 \mu \mathrm{m} \times 200 \mu \mathrm{m}$, and twice as long in the outer two. The middle region consists of 3 layers of short strips (SS) with length $2.5 \mathrm{~cm}$ and pitch $80 \mu \mathrm{m}$, out to a radius of $60 \mathrm{~cm}$ and $|z|<100 \mathrm{~cm}$. The two outer layers use long strips (LS) of the same pitch but length $9 \mathrm{~cm}$. The inner radii of the endcap disks are defined to provide coverage out to $|\eta|<2.5$. Each of the SS and LS sensors consist of two layers, with the strips rotated with respect to one another, thus providing three-dimensional information. The sensors in all three subsystems overlap slightly to eliminate gaps in solid angle coverage.

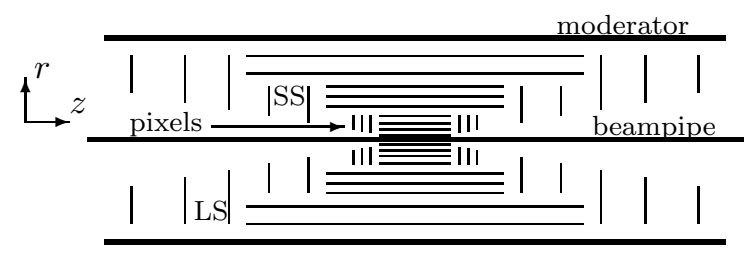

Figure 2. "Strawman" Inner Detector layout, showing the position of the pixel barrels and endcaps around the center, surrounded by the short strips and then the long strips. Sensitive layers are denoted by thin lines. The beampipe (center) and moderator (top and bottom) are shown as thicker lines.
Compared with the current ATLAS detector, the SS barrels correspond roughly to the current Silicon Tracker (SCT), and the LS barrels replace the TRT. The innermost SCT layer, however, has been replaced by a pixel layer, and the innermost pixel layer will either move farther away from the beam, or, as mentioned above, be replaced by a more radiation-hard technology.

A program of performance evaluations has already begun, using both parametric and Geant4based simulations. Initial results from the Geant4 simulation suggest that the occupancy of the innermost SS layer (at $r=38 \mathrm{~cm}$ ), due only to the pileup events, is approximately $2 \%$, which is already above the conventional "rule of thumb" of $1 \%$ for efficient pattern recognition with an acceptable fake rate. Further studies using physics signatures such as $t \bar{t}$ and $Z^{\prime} \rightarrow \ell^{+} \ell^{-} / b \bar{b}$, at different $Z^{\prime}$ masses, should illuminate whether the resulting pattern recognition performance will be acceptable for ATLAS physics, both inside and outside of jets. The ability to distinguish $b$ quark from light quark jets will also be investigated.

Variations on the strawman will be studied to optimize the layout. These include, for instance, the omission of stereo information at different strip layers, and the addition of layers and disks; in particular, additional LS layers may be needed for the reconstruction of late secondaries. Building SS and LS barrels to the same length has been suggested in order to simplify the engineering and servicing of these detector subsystems. Another interesting engineering-related possibility is to construct the endcaps in the shape of cones rather than disks. A "double $b$ layer" has also been suggested, with an inner layer closer than $5 \mathrm{~cm}$ and an outer layer $1 \mathrm{~cm}$ farther out, the two layers providing redundancy for one another.

There remains, however, significant uncertainty as to the actual nature of pileup events at $\sqrt{s}=14 \mathrm{TeV}$, as well as the actual material profile of the current detector with its sensors and services. LHC data will help reduce these uncertainties. One of the objectives of the current simulation program is therefore to provide a natural means to incorporate LHC data as it becomes available, and to accommodate the evaluation of layout options as late in the design phase as pos- 
sible.

Another issue is the need to simplify the engineering of the Inner Detector upgrade, especially given the tight timescale for its design and construction. It is essential that the simulation project remain in close contact with groups working on engineering, services, and readout.

\section{Schedule}

It is expected that installation of detector upgrades will begin in late 2014 in order to be ready for the resumption of data taking in April 2016. This is already an aggressive schedule, and does not yet accommodate the anticipated timescales associated with opening the endcap cryostat for calorimeter upgrades. These timing issues are still being explored.

A Technical Design Report is scheduled to be ready in 2010 to allow sufficient time for production readiness reviews later that year and the subsequent above-ground construction phase. The design phase is therefore already in progress, with a strawman layout for the ID (as a starting point for design iterations) defined in late 2006. Details of a $b$ layer replacement, scheduled for 2012, are also being worked out.

\section{Conclusion}

It is evident that the SLHC will pose a significant challenge to the ATLAS experiment, with its aggressive timescale and harsh environment. It is anticipated that the entire Inner Detector will be replaced, probably with an all-silicon tracker; major upgrades are also being considered for the forward calorimeters, muon chambers, and beampipe. The recent narrowing of possible machine scenarios has helped focus plans, as will the imminent arrival of LHC data in the current detector. In light of these challenges - and considerable physics opportunities - a number of $R \& D$ efforts have already begun in order to be ready to take SLHC data in 2016.

\section{Acknowledgements}

The author wishes to thank Nigel Hessey, David Lissauer, Ian Dawson, Gerald Oakham, and the ATLAS upgrade community for their useful comments and contributions to this report. He also thanks the Symposium organizers for arranging a thoroughly enjoyable conference.

\section{REFERENCES}

1. The ATLAS Collaboration, "ATLAS Detector and Physics Performance: Technical Design Report", CERN/LHCC 99-14, 25 May 1999.

2. W. Scandale, these proceedings.

3. J. Nash, these proceedings; G. Azuelos et al., $J$ Phys G28, 2453 (2002).

4. F. Gianotti et al., Eur Phys J C39, 293 (2005).

5. S. Tapprogge, ed., "Outline of $\mathrm{R} \& \mathrm{D}$ activities for ATLAS at an upgraded LHC", CERNATL-COM-GEN-2005-002, 23 Jan 2005.

6. R. Garoby (PAF), J. Ellis (POFPA), "Re: Towards a preferred bunch-spacing scenario for the SLHC", memo to R. Aymar and J. Engelen, 20 Dec 2006.

7. O. Kortner, "MDT performance and new detectors", Muon Detector Upgrade Workshop, CERN, 25-26 Jan 2007.

8. V. Hedberg, "Beampipe and shielding for the ATLAS upgrade", ATLAS Upgrade Workshop, CERN, 1-2 Oct 2006.

9. F.G. Oakham, "Temperature rise in the FCal at $10^{35}$ design luminosity", ATLAS LArg Calorimeter Upgrade Workshop, CERN, 4 Dec 2006.

10. I. Dawson, "Radiation predictions at the SLHC and irradiation facilities", ATLAS Tracker Upgrade Workshop, Liverpool, 6-8 Dec 2006.

11. C. Da Via et al., Nucl. Inst. Meth. A509, 86 (2003).

12. H.G. Moser et al., "R\&D for a novel pixel detector for SLHC", ATLAS Tracker Upgrade Workshop, Liverpool, 6-8 Dec 2006.

13. M. Campbell et al., Nucl. Inst. Meth. A560, 131 (2006).

14. W. Adam et al. (The RD42 Collaboration), Nucl. Inst. Meth. A565, 278 (2006). 